\title{
Replacing De(ad)scriptive Geometry...
}

\author{
Axel Pavillet \\ axel.pavillet@polytechnique.org
}

\begin{abstract}
CAD tools are available for designing 3-D parts. They can also be used as pure geometric tools.

$D G$ does not efficiently use CAD tools and could be replaced with an experimental geometry based on theorem verifications and $3 D$ geometric constructions.

Theorem verifications are a shortcut for what the student did not learn. Using CAD software, geometric constructions in space are not purely theoretical. They become as real as constructions in plane geometry and make Solid Geometry closer to an experimental science.

Increasing the productivity and usefulness of a graphics course without diminishing its educational value, experimental geometry makes it possible to teach more geometry, more quickly to more students.
\end{abstract}

\section{INTRODUCTION}

Contrary to popular belief, Descriptive Geometry (DG) is not a science. It is a two hundred-year-old tool created by Gaspard Monge. Two arguments can be given for this fact. The first one is that no new theorem or geometric property has ever been discovered by using DG [1] (p. 285) though Monge used it a lot to demonstrate geometric properties [1] (pp. 195-202). One can suspect that he did so in order to promote his new tool (using the word marketing here would be an anachronism!). The second argument is that DG was for twenty years a French military secret [2]. Science, even nuclear physics, never has, never will be a secret per se. Only technologies and tools used by, or developed with, science are classified.

Because it is a tool, DG can become obsolete, and to prove this fact we only need to show that we can get all of the results of DG through a quicker, more efficient (and less painful) way. Make no mistake, the goal here is not to promote the teaching of analytic geometry in three dimensions at the expense of DG. Nor is it to promote verbal thought at the expense of the mind's eye as define by E.S. Ferguson [3]. On the contrary: in his 1977 article Ferguson not only underlines the importance of graphics in the most general sense but he also forewarns us of the danger of neglecting graphics in favor of numerical computation, particularly in engineering colleges. The best way for graphics to lose this competition would be to remain static.

\section{AN EXAMPLE OF Reverse Descriptive Geometry}

We start with an example coming from the design of a new algorithm in computational geometry. In this case graphics and computation are not in competition but rather complementary.

We recall first that a Voronoï diagram (VD) for a set of sites in the plane partitions the plane into regions called Voronoï sectors, one to a site, so that any point in the region containing a site is closer to that site than to any other site. In well-behaved cases, the limit between two sectors is a curve called a bisector. If the bisector is part of a cartesian oval [4], the VD is said to be a compoundly weighted VD [5]. In order to design a circular sweep algorithm for such a Voronoï diagram it was necessary to draw, easily, numerous arcs of cartesian ovals [6]. Here to draw is a key word. Obviously it would be a catch-22 situation if you had to compute them in order to draw figures to design the algorithm. Unfortunately no software directly gives such a possibility (a circle is defined by three conditions, a cartesian oval is defined by six [7]). However using Quételet's theorem (1823) [8], one can draw a cartesian oval as the horizontal projection of the curve of intersection of two cones of revolution with parallel, vertical, axis.

3D CAD tools are widely used for the design of 3-D parts, but they can also be used as pure geometric tools. Using such software, it was possible to draw the numerous figures necessary for the basic understanding and design of a sweep-cone algorithm for these types of VD.

Now, there are two points to be made here. The first one is that it might not have been possible to design such an algorithm without being able to draw figures. This is Ferguson's idea: there is more in a figure than in a numerical computation. The second one is that a cartesian oval is a plane curve; the VD diagram is in the plane but all the figures were done in space with a $3 \mathrm{D}$ CAD software, the useful results being obtained by using one and only one of the projection. This is reverse descriptive geometry, but then if we use a 3D CAD software to get results in the plane, why would we go on using DG to get results in space?

\section{DG DOES NOT MAKE AN EFFICIENT USE OF CAD TOOLS}

Descriptive geometry was designed for the exclusive use of ruler and compass; CAD tools uses much more powerful hidden numerical computation. Obviously a valid solution to a geometric problem given by DG will stay valid with CAD software. However there is no reason why an optimum method in DG would stay optimum when transferred to CAD software.

The drawing of a curve in DG, but also the development of a single ruled surface, use two principal methods: the descriptive method and the geometric method [9] (p. 54-55).

A solution using the geometric method will determine principal geometric elements of the curve or surface to be drawn and draw it from there: for example, using geometric theorems, one will find the center and one point of a circle to be drawn then draw it from there with a compass.

Descriptive method will directly and repeatedly use the given data of the problem; for example to draw an ellipse given by a point and its two foci, one will draw as many points as necessary by intersecting two arcs of circle centered on the given foci, the sum of the radii of those two circles always being equal to the sum of the distances to the given point from each focus. 


\section{A. "Avoid drawing the ellipse"}

With this latter example we can pursue the idea of how to use an elliptic compass (more generally a conic compass).

Treatises of DG are not really treatises, they are rather a compendium of solutions to various types of exercises; one of the best summa in this field is the book by F.G.M. [9], about 1700 pages in two parts. In this book, like in all its kind, there is a kind of leitmotiv which is avoid drawing the ellipse: every time you have two solutions to an exercise, one where you have to draw a conic and one where you have a workaround with ruler and compass, the workaround is considered to be the optimum solution, and very rightly so, $\ldots$ at least as long as you cannot use an elliptic compass. For example in F.G.M.'s book, the solution to the problem \#449 p. 588 states: it is preferable to avoid drawing the ellipse, because this construction can only be done by points and in an approximative manner. In any case it is not always possible to avoid drawing the conic and of course nobody has, ever, drawn a circle by points using a descriptive method, except out of ignorance [2] §2.6.1!

Obviously today with CAD software, if you can use a geometric method to draw a conic, the precision of your solution will be as good as the solution with ruler and compass. It might also be as quick, or even quicker, as the workaround, sometimes very tortuous, given by the treatises.

Yesterday's optimum is not optimum anymore and we have to examine ways to use the conic compass with CAD software.

Being a conic passing through both circular points, a circle is determined by three conditions and the real circular compass can be used only when you have the center and the radius or the center and one point of the circle to be drawn. The CAD circular compass of any 2D CAD software is indeed better because it gives you other natural choices to draw a circle. You can use a diameter, and of course you have the more interesting choice of three points with the dual choice of three tangents and any combination of points and tangents. Therefore, because you don't need an intermediate construction to draw the circle circumscribing a triangle, the CAD compass is more efficient than the real one.

For an elliptic compass things are more difficult. Real physical elliptic compasses have never been more than a geometric curiosity [10] or gardening tools [11]. Elliptic compasses based on CAD software are much more interesting. Of course they can, or could, as easily deal with hyperbolas; they are really conic compasses even if historically the term elliptic compass should stay. The gap with CAD circular compasses is important. On the one hand the conditions that define an ellipse are more numerous: five instead of three. On the other hand, the choices given by the software to draw an ellipse are more limited than for a circle (mainly based on minor and major axis). With Autocad 2002, even the basic choice of two foci and one point is not offered; neither is the direct drawing of a conic defined by any combinations of five points or tangents (with Rhino $3 \mathrm{D}$ the choice of a one point and foci is offered for ellipse and parabola).

In spite of these limitations, especially if you cannot avoid drawing the ellipse, the use of the elliptic compass is very interesting if the student knows how to construct the axis and vertices of an ellipse given by five arbitrary points. This is equiva- lent to the construction of the circumscribed circle of a triangle but is not part of any DG course (it is usually part of projective geometry [12]). Anyway, most cases are easier because usually some basic elements of the ellipse are already known: axis, foci, conjugate diameters, etc.

Therefore, the elliptic compass is a basic tool which is underused because of both DG traditions and the fact that the various geometric methods necessary to use it and replace the descriptive methods have not been taught to the students.

\section{B. Development of a single curved surface}

From the former example we should not deduce that only geometric methods can be improved by CAD software and that a conversion is needed only for descriptive methods. Even with descriptive methods, CAD software can do more.

The development of a single curved surface can be done by the geometric method if the surface is as simple as the frustum of a cylinder, for example. However if the surface is more complex one should resort to the descriptive method, which can be a very lengthy process.

With a 3D CAD software like Autocad 2002, one starts by drawing the surface in space. Then, as in DG, the surface is decomposed in planar elements but because it is done in space, it is done only once and then the planar elements are aligned on a plane one by one and side by side. The operation is much quicker than in DG and at least as precise.

Let's follow a detailed example (Fig. 1), using Autocad 2002 instructions, of the development of the frustum of an elliptical cone comprised between two concentric spheres centered on the vertex of the cone (therefore the curves which limit the frustum are spherical conics [9] (pp.754-756)). $\Gamma$ is the initial 3D surface. Using the instruction Divide, a set of regularly spaced points is added to one of the spherical conic. Based on these points we derived the surface $\Gamma^{\prime}$ formed by the 3D faces approximating $\Gamma$. Then the first quadrilateral $A B C D$ is transferred on the horizontal plane using the instruction Align. The following quadrilateral is then aligned again by taking care of using point $b(c)$ as the destination point for the source point $B$ $(C)$ of the quadrilateral $B E F C$ (a third point can be taken anywhere on the horizontal plane). The result can be printed, cut or stamped out. One sees here that the development follows the descriptive method but is done directly from space. For a quick prototyping this is also much quicker than the precise numerical computation and drawing of this surface with a tool like Maple. Of course, if you use Rhino 3D (Fig. 2) you don't even need to use the descriptive methode. One instruction is sufficient: unroll developable srf will do all the work for you. We will examine later the problem of software dependency.

\section{EXPERIMENTAL GEOMETRY}

Those two examples are sufficient to show that there is a huge difference between what is taught and what can and should be done with CAD software. Obviously, it is the intellectual value of DG as a training exercise for an engineering student which has justified keeping the course in the curriculum. It may remind the oldest readers of another interesting debate about the teaching of Latin in high school. In a way, we try to teach Latin 


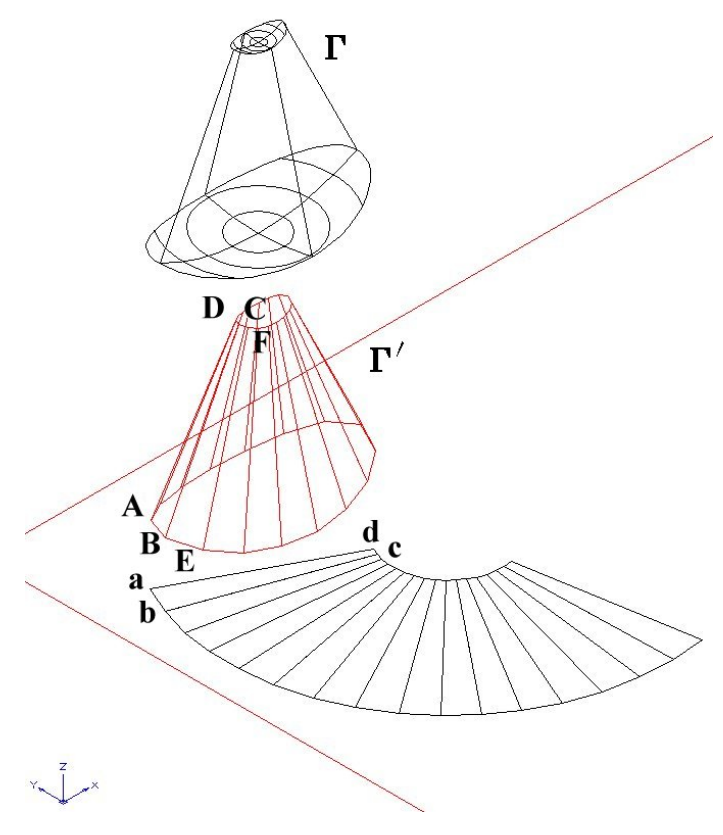

Fig. 1. Development from space - Autocad

as a modern language to a generation of students trained with video games. No wonder, in Universities where the graphics course is still taught, it is unlikely to be the most popular course among the students.

If we go back to our two examples, elliptic compass and surface development, we see that the second one shows something more: the work can be done directly in space. This brings us back to our very first question: if we can work directly in space do we really need to teach DG? The parallel with Latin implies immediately a second one: is there a way to keep the intellectual and educational value of DG and teach something really useful at the same time, a trade-off between the position of Ferguson, but also the position of Stachel in [13] and the resignedness of Sakarovitch [1] (p.354)?

The following proposal, replacing DG by a kind of Experimental Geometry has been given a name which is voluntarily provocative but shows the necessity of compromising between pure science and practical training. This is because it seems difficult to keep the rigorous aspect of a theoretical science when so little geometry is taught nowadays at the secondary level. The gap cannot be filled that easily at the engineering undergraduate level and we may have to choose between experimental geometry and no geometry at all.

Experimental Geometry would be based on two main concepts: theorem verification and geometric constructions in space.

\section{A. Theorem verification}

Obviously theorem verification is a last-resort solution. No one can really consider that avoiding theorem demonstration is a good solution; however theorem verification is not something new. One can find it in a text of Sylvester of 1866 about the intersection of three cones: ... The verification of these laws of intersection might be used to form the subject of a new and
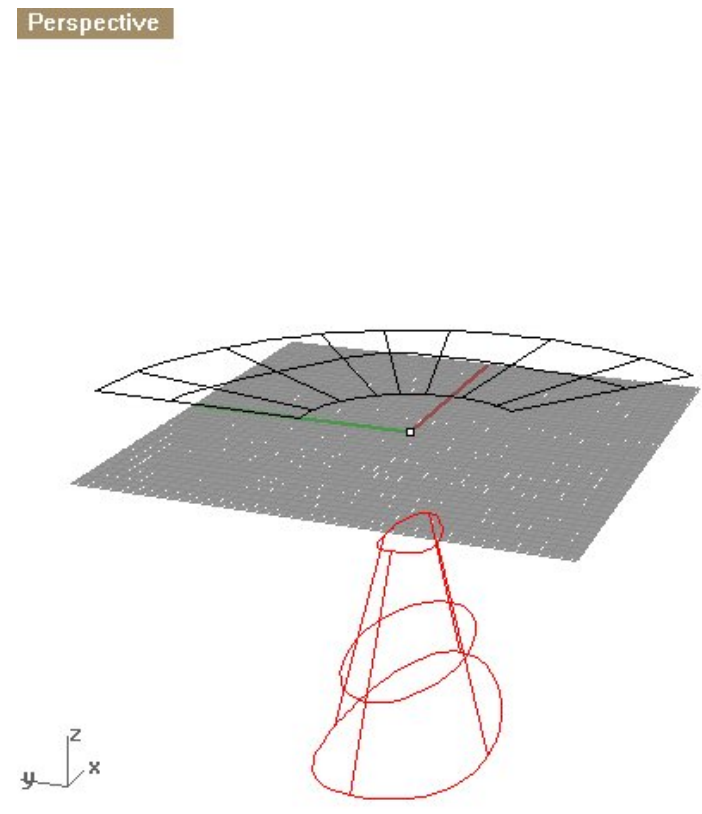

Fig. 2. Development from space - Rhino 3D

instructive plate for students of ordinary descriptive geometry. [14]. The interesting idea behind this citation is that there will always be more people able to verify a theorem than people able to demonstrate it.

But how can we actually verify a theorem of solid geometry (SG)?

\section{B. Geometric constructions in space}

As with DG, CAD software bring something very new to the principle of geometric constructions in space. Geometric constructions in plane geometry have always been part of the teaching of elementary geometry. Mathematics might be abstract but constructions in plane geometry are not. You do them with ruler and compass: the theory is put to practical use. On the contrary, geometric constructions in space were not, as we can read from [15] by Altshiller-Court who has probably written one of the last books exclusively dedicated to SG:

Geometric Constructions in Space. When speaking of constructions in space we assume that we are able (a) to construct a plane given three of its points not lying in a straight line; $(b)$ to construct the line of intersection of two planes; (c) to construct the point of intersection of a line and a plane; (d) to carry out all plane constructions in any plane in space. These constructions are purely theoretical, for we have no practical method to actually carry them out. However, in Descriptive Geometry all these constructions are executed by ruler and compasses.

It is clear that you can make constructions in space with DG, but what they really are, are constructions in plane geometry. The first edition of Altshiller-Court book's is from 1935 and at that time he was right to write that: These constructions are purely theoretical, for we have no practical method to actually carry them out, but let's see if this phrase could be written today. 
First with regards to the tools, the first one, to create a plane from three points, is available from any 3D CAD software, with an obvious restriction which is the fact that it is not an infinite plane, but what is called a 3D face in Autocad 2002 can always be extended. The second and the third one (construct the line of intersection of two planes or one line and a plane) are directly available in Rhino 3D and though they are not directly available in Autocad 2002, there are easy work-arounds. Of course, where the result is a line segment, a straight line can easily be drawn through the extremities of this segment. Finally the possibility of carrying out any $2 \mathrm{D}$ constructions in any plane is present for any 3D CAD software.

Therefore, we have all the tools ready to work directly in space. Of course one can object that we are drawing on a computer screen and therefore we are drawing on a plane. However perspective views and $3 D$ orbit in Autocad or Rotate view with Rhino 3D make space really available even on the computer screen. We can actually look at the constructions in space and feel them in the same way we do it with ruler in the plane; there is no need to wait for 3D Displays [16].

None of those softwares are perfect. They have not been designed to teach solid geometry; the objects, with the exception of the straight line in Autocad, are not infinite; there is no easy way to draw using points at infinity, i.e. directions, and the list of objections to the use of a CAD software as the rule and compass of SG could be extended at will.

But it does not matter; the fact is that we can actually carry those constructions in space and that the Altshiller-Court statement is now obsolete. Besides, we can make them with an increased productivity if, in the plane we use an elliptic compass and if in space we use the possibility of drawing basic surfaces (or volumes) as a sphere with various choices, the minimum being center and one point or a cone with vertex and directrix. These tools, sphere generator (the expression "spherical compass" is already taken) and cone generator (to which we may have to add the torus generator) are really the equivalent in space of the plane circular compass, Altshiller-Court tools being equivalent to the ruler. In the plane, the use of the circular compass is not compulsory, every construction can be done with the ruler only but it increases productivity. In space, the use of a spherical compass will not really change the difficulty of the construction but will make it possible to do it much faster, thus avoiding numerous auxiliary constructions and the use of the descriptive method. More time will be spent learning than drawing.

To develop an example would be too long for this article, but the interested reader can verify that three cones of revolution with non coplanar, parallel, axis have four concyclic points of intersection. Furthermore, varying the angle between two coplanar generators of one of the cones, the planes defined by the four points of intersection of the two fixed cones and the variable one will belong to a pencil of planes (see [6] for details). This example will use all the tools defined above. It will also use most of the basic instructions of a 3D CAD software. Finally, one can try to draw the same figure in DG (Fig. 3)!

This example would underline one more technical but essential point. Going back to the book of Sakarovitch [1] (p. 216) there is a very interesting citation from Chasles (1837): The old geometry is filled with figures. The reason is simple. Since we were missing general and abstract principles, every issue could only be solved concretely, using the very figure which was the object of this issue, only its view could help discover the elements necessary to the demonstration or the solution we were looking for. But we experienced the drawbacks of this methodology due to the difficulties of construction of some figures and through their complication which makes their understanding laborious and painful. It is mainly for the problems of solid geometry, in which figures can become really impossible that the drawbacks we underline are felt the most.

A 3D CAD software gives us the tools of solid geometry. Not only has it enabled us to do constructions in space but it has also brought something more: the layer concept. The use of layers that can be switched on and off greatly facilitates the understanding of a figure, which was the second reason given to explain the difficulties of using figures in space. Adding that to an easy use of colors, would Chasles write the same thing today?

We should not forget that DG was in its time a revolution due to Monge's genius but also was the result of the standardization and normalization of various methods already known. It is possible that the use of engineering software to teach SG may need the same effort of standardization to reach maximum efficiency, but even without it there is already a huge improvement over classical DG.

When it became algebraic, geometry lost its appeal for engineering teaching even though solid geometry will always be an essential part of mechanical or civil engineering. The possibility of carrying out real constructions in space has to be considered as a golden opportunity either to bring this teaching back where it disappeared or to update it where, by maintaining DG in the curriculum, it became obsolete.

\section{Software dependency}

We have already seen an example of software dependency with the development of a single ruled surface. Reliance on software instructions to teach geometric concepts might be tricky because new versions make some exercises obsolete when the construction is embedded in the software. The example of surface development shows that if you use Rhino 3D the exercise will not be very useful for a student: there is nothing left to learn! On the contrary if you use Autocad, the result is quicker than with DG and the concept of development is learned. Teaching classical DG with software as powerful as Autocad, Rhino 3D or ProE is not a good solution because the 3D capabilities are underused (even the elliptic compass in the plane as we have seen) and the student knows it. He may not know how to solve the DG problem with the 3D CAD software but he knows something is wrong. To teach DG, a 2D CAD software as the freeware QCAD 1.5 is more than sufficient and, because this software does not even have an elliptic compass, there is no contradiction between what is taught and what can be done.

This principle of no contradiction between the possibilities of the software used and what is taught should be followed for all software employed during a course. Ideally one would wish 


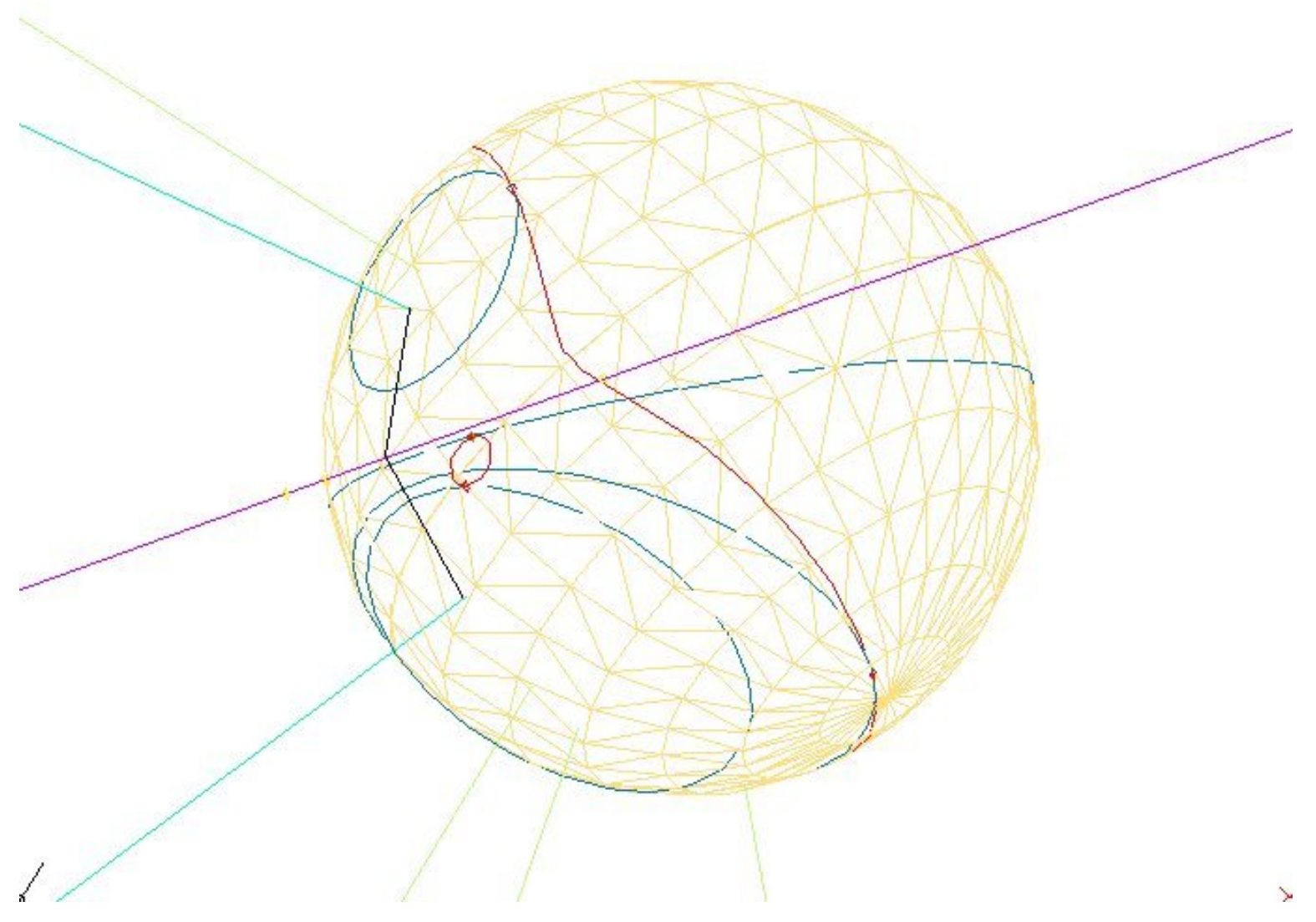

Fig. 3. Curve of intersection of two fixed cones (red). Pencil of circles supporting the points of intersection with a third varying cone (blue)- axis of the pencil of plane (magenta)

to have an open source freeware dedicated to teaching experimental geometry. This would automatically ensure the standardization of tools and the lack of contradiction. However in the foreseeable future using 3D CAD engineering software will stay the only realistic solution.

As we have seen the software used to solve a construction problem should be powerful enough to allow for a quick solution, saving most of the material work (in effect avoiding as much as possible a descriptive method). It should not, however, be so powerful as to embed all the intellectual work within the software. This will lead to the need to use more than one CAD software during the course which is not a bad thing per se.

There is a caveat here. Software editors are very interested in having their software used in university training for a very simple reason: natural laziness, of course, but also that the need for immediate efficiency makes even bright students and engineers reluctant to switch software once they know one. This is true for any software but it does apply to a greater degree to software with a steep learning curve. University or student prices are not a gift, they are an investment. Therefore low licensing fees will very often come hand in hand with an exclusivity clause. The difficulty of convincing a university administration to buy and maintain various versions of competing softwares should not be underestimated.

Satisfying this requirement will ensure that neither the course nor the student would be software dependent. Moreover, the professor will have to adjust his course material to every new issue of the software used. Very few DG books have been pub- lished in the last twenty years. DG courses were very comfortable for professors because they did not not need frequent upgrades; the course will not be static anymore.

\section{Prospective Syllabus}

A prospective syllabus for this experimental geometry course would in fact follow loosely the syllabus of a DG course.

The introduction would include a definition of the geometric elements: points, straight lines, planes and their projective properties; the definition of elements of differential geometry and the definition of the tools of SG as defined by AltshillerCourt.

To replace the chapters of DG dealing with parallelism, perpendicularity, intersection of planes, dihedral angles, tetrahedron geometry would be taught. This would be particularly interesting because the construction of the geometric elements of a tetrahedron (centroid, Monge point, Euler line, etc.) will obviously require the use of the basic tools of SG, but also because the students will concretely see or discover that triangle geometry (plane geometry) cannot be extended to tetrahedron geometry (SG) without precautions: it is easy to verify that most students believe that every tetrahedron has an orthocenter.

Sphere geometry would be next. With the use of the sphere generator, productivity would be good enough to enable covering a wide view of spheres' properties from the spheres linked to the tetrahedron to a pencil or network of spheres.

For this first part of the course, Altshiller-Court's book would be a good guide. 
Quadrics would naturally follow. The study would encompass ruled but also double curved quadrics. Here a good guidance would be the book by Rouché and de Comberousse [11] (Livre VIII).

Some time should also be dedicated to the torus and the helicoid which are essential surfaces for the mechanical engineer. Finally to keep with the descriptive method, it could also include drawing wireframe surfaces as cyclides or developables with CAD software.

At the end of this syllabus, the engineering student will have met the same intellectual challenges he would have got with classical DG but he will also have learned much more geometry than before, and now this knowledge will be a useful one for her or his professional life.

\section{A. Time allocation}

Time allocation for this course would be generated by drastically shortening not only the teaching of classical DG where it has been maintained but also the teaching of engineering drawing. In DG, distance between two lines (which was the most basic application of DG), true view, auxiliary view, rotations, etc. are totally obsolete. In engineering drawing, teaching should be limited to basic principles now that its most fastidious part, normalization, is software embedded and does not really need to be burnt into the students memory.

\section{B. Teaching space}

Engineering students, especially in mechanical or civil engineering, are not aiming at abstract science; so teaching SG centered on these two concepts, theorem verification and constructions in space and applying such a syllabus should not be a problem for the students or for their subsequent employers. With this orientation, Solid Geometry becomes closer to an experimental science than to a theoretical one.

Moreover, Sakarovitch in [1] (p. 279) recalls that DG was created in the revolutionary France of 1794 to improve the yield of teaching space to the scientific engineering elite needed by the young republic. From what is said before, it is clear that today DG is more a liability than an asset when it comes to teaching space to an engineering student of the Nintendo generation. Because experimental geometry would improve the yield of teaching space and be accessible to more students than a purely theoretical science, it would be a good way to replace DG without suppressing geometry of the curriculum. Moreover there is a gaming side to geometric constructions with 3D CAD software which would make it more acceptable to students than drawing 2D principal views with the same software.

\section{CONCLUSIONS}

We conclude with some ideas about the finality of teaching geometry to mechanical engineering students.

Before the 1970s engineers had a good knowledge of geometry, but no efficient tools such as CNC machining to realize complex mechanical parts. Geometry teaching is out of date; so now they have the tools but not the knowledge, at least upon graduation. If the engineer is in the Aeronautic industry, it is not really a problem because the industry has the knowledge he should have learned in University, but if he is the sole engineer of a small business, he may well overlook an optimum or innovative solution.

Similarly, the cost of a mechanical part is a function of the time spent machining it, but the correlation between the complexity of its geometrical shape and the time spent on machining is much weaker than before. The cost of using an ellipsoidal shape in a design is no different today from the cost of using a spherical one. The use of a double curved surface instead of a single curved one is not as bad as before. It is always better to have a simpler design but if it is impossible the engineer mastering geometry would obviously have an edge.

We can admire the exceptional longevity of DG as a tool as very few tools have been that persistent. However, the fact is that, as a tool, DG is obsolete. Finally, SG, the science, remains more pertinent than ever. Today's teaching should use the new software tools to teach more geometry more quickly to more students.

\section{ACKNOWLEDGMENTS}

The support from the NSERC Design Engineering Chair at McGill University, Design for Extreme Environments, is dutifully acknowledged.

The design of the sweep-cone algorithm was done as part of the author's PhD thesis written under the supervision of Prof. Timothy Walsh, Department of Computer Science, UQAM. The author also wishes to thank Prof. Walsh for style editing this article.

\section{REFERENCES}

[1] Joël Sakarovitch Épures d'architectures, de la coupe des pierres à la géométrie descriptive XVI $I^{e}-X I X^{e}$ siècles, Birkhäuser, Basel,1998

[2] Boris Asancheyev, Épures de Géométrie Descriptive Concours d'entrée à l'École Normale Supérieure, Hermann, Paris, 2002, pp. 11-13

[3] E.S. Ferguson, The mind's eye: non verbal thought in technology, Science, vol. 4306, 1977, pp 827-836.

[4] Francisco Gomes Teixeira, Tratados de las curvas especiales notables. Memorias de la Real Academia de Ciencias Exactas Fsicas y Naturales de Madrid, Tomo XXII , Madrid, 1905, pp 157-171.

[5] A. Okabe, B. Boots and K. Sugihara, Spatial Tessellations, John Wiley \& Sons, Chichester, England, 1992, pp. 136-137.

[6] Axel Pavillet, "Une famille d'algorithmes à balayage circulaire pour le calcul de diagrammes de Voronoï de points ou de cercles pondérés", Thèse de doctorat en mathématiques, Université du Québec à Montréal, Qc, 2004, ch.10-11 - Results to be published.

[7] J.J. Sylvester, Mathem. Questions from the "Educational Times", Miller, London, 1866, question 1990 .

[8] Paul Baudoin, Les ovales de Descartes et le limaçon de Pascal, Vuibert, Paris, 1938, ch. 9.

[9] F.G.M., Exercices de Géométrie Descriptive, J. Gabay, Sceaux, France; 1996 (reprint from 1893).

[10] George Salmon, A treatise on conic sections, Chelsea Publishing Company, New York, 1962, pp. 218-219 (reprint from 1879).

[11] Eugène Rouché, Charles de Comberousse, Traité de Géométrie, J.Gabay, Paris, 1997, pp. 289-290 (reprint from 1900).

[12] G. Papelier, "Géométrie projective. Application aux coniques", Exercices de Géométrie moderne, Tome IX, Vuibert, Paris, 1958, pp. 107-119.

[13] Hellmuth Stachel, "What is Descriptive Geometry for?", in Proceedings Dresden Symposium Geometry DSG.CK 2003, Dresden, Germany, 2003.

[14] James Joseph Sylvester, "Supplemental note on the analogous in space to the cartesian ovals in plano", Philosophical Magazine, Vol. XXXI, London, England, 1866, p. 560.

[15] Nathan Altshiller-Court, Modern Pure Solid Geometry, Chelsea Publishing Company, Bronx, NY, 1964, pp. 1-2.

[16] Martyn Williams and Tom Krazit, "Will Your Next Display Be 3D? "IDG News Service. PC World.com - Yahoo News, Fri May 7 2004, 9:00. 